\title{
Ultrasensitive detection of endotoxins using computationally designed nanoMIPs
}

Zeynep Altintas $^{\dagger a, b_{*}}$, Mohammed J. Abdin ${ }^{\dagger a}$, Alexander M. Tothill ${ }^{\mathrm{a}}$, Kal Karim ${ }^{\mathrm{a}, \mathrm{c}}$, Ibtisam E. Tothill $^{\mathrm{a} *}$

${ }^{\text {a }}$ Cranfield University, Cranfield, Bedfordshire MK43 0AL, England, UK

${ }^{\mathrm{b}}$ Technical University of Berlin, Straße des 17. Juni 124, Berlin 10623, Germany

${ }^{c}$ University of Leicester, Leicester LE1 7RH, England, UK

*Corresponding authors:

Z. Altintas

I.E. Tothill

Tel: +44 (0) 7500766487, Fax: +44 (0) 1234758380

E-mail: zeynep.altintas@ cranfield.ac.uk

i.tothill@cranfield.ac.uk 


\begin{abstract}
Novel molecularly imprinted polymer nanoparticles (nanoMIPs) were designed for endotoxin from Escherichia coli 0111:B4, using computational modeling. The screening process based on binding energy between endotoxin and each monomer was performed with 21 commonly used monomers, resulting in the selection of itaconic acid, methacrylic acid and acrylamide as functional monomers due to their strong binding interaction with the endotoxin template. The nanoMIPs were successfully synthesized with functional groups on the outer surface to aid in the immobilization onto sensor surface. The solid phase photopolymerization approach used for the synthesis of nanoMIPs ranging from $200-235 \mathrm{~nm}$ in diameter. The limit of detection and $\mathrm{K}_{\mathrm{D}}$ were significantly improved when endotoxin samples were prepared using a novel triethylamine method. This improved the efficiency of gold nanoparticle functionalization by targeting the subunits of the endotoxin. Compared to the vancomycin MIP control, the endotoxin MIPs displayed outstanding affinity and selectivity towards the endotoxin with $K_{D}$ values in the range of $4.4-5.3 \times 10^{-10} \mathrm{M}$, with limits of detection of $0.44 \pm 0.02 \mathrm{ng} \mathrm{mL}^{-1}$ as determined by surface plasmon resonance (SPR) sensor when itaconic acid was used as the functional monomer. The MIP surface can be regenerated > 30 times without significant loss of binding activity making this approach highly cost effective for expensive analyte templates. The combination of molecular modeling and solid phase synthesis enabled the successful synthesis of nanoMIPs capable of recognition and ultrasensitive detection of endotoxins using the highly sensitive SPR biosensor with triethylamine method.
\end{abstract}

Keywords: Molecularly imprinting polymers (MIPs), Endotoxins, Computational modelling, Nanomaterials, Triethylamine, Biosensor 


\section{Introduction}

Endotoxins also termed lipopolysaccharides (LPS) are found on the cell walls of most gram-negative bacteria and some cyanobacteria. The endotoxin structure is composed of three regions: the outer polysaccharide, the core oligosaccharide and the lipid A region. The biological active unit is the lipid A and is called the "endotoxic principle" [1-3]. Endotoxins pose significant health risks to humans through intravenous and inhalation exposure. The biological activity of lipid A in humans and other species is potent and wide-ranging. Some of these health effects range from mild fever, respiratory, gastrointestinal disorders and sepsis [4, 5]. Serious reactions to LPS exposure from hemodialysis are well known and tightly regulated; in addition endotoxins in drinking water pose a potential concern [6-8].

There is a need for novel affinity ligands that can demonstrate high affinity and selectivity towards endotoxin that can be used to enhance current endotoxin removal and detection techniques. The inherent expense and difficulty in raising antibodies towards endotoxin which require the use of animals [4, 9] and the neurotoxic effects of polymyxin B [10-12] has confirmed the need for the application of other technologies in the production of affinity ligands towards endotoxins. This provided an opportunity to design and synthesize molecularly imprinted polymers (MIPs) as artificial sensing ligands for endotoxin capture and detection.

In this work, MIP technology has been combined with a computational approach using a molecular modeling program to specifically design artificial receptors that target the conserved region of the endotoxin structure. The polysaccharide chain of the endotoxin can vary significantly within species of gram-negative bacteria, whereas the lipid A and inner core of the oligosaccharide chain display a more conserved region [13]. The molecular imprinting of 
endotoxin is very difficult due to its large molecular weight $(10,000-20,000 \mathrm{Da})$ and its amphiphilic (both hydrophilic and hydrophobic) characteristic. Our solid phase approach allows the covalent attachment of the endotoxin to silica beads for molecular imprinting to occur. The molecular imprinting technique is based on a complex formed between the template and functional monomer(s) by self-assembly. Strong interactions between the functional monomers with the target allow producing MIPs with greater stability, which in turn improves the selectivity of the MIP cavity towards the target [14]. The composition of polymers was traditionally selected through literature or experimental trials, whereas the computational approach allows use of a virtual library containing the 21 most commonly used monomers. The selection of monomers is based on the strength of interactions with the template and simulated annealing is used to analyse the arrangement of monomers around the endotoxin template to determine the optimal MIP composition [15].

The MIPs are synthesized using the solid phase synthesis procedure [16-18]. This involves covalent immobilization of the template on to glass bead supports to allow the formation of the MIP around the immobilized template and the process of separating low affinity and high affinity MIPs from the template easily and efficiently while preventing from template leaching. A key feature of this procedure is the ability to make post modifications without affecting the binding site. As MIPs are formed around the template the iniferter moieties are still present on the outer surface allowing functional groups to be grafted on to the surface of the MIPs without modifying the cavity. The combination of molecular modeling and solid phase synthesis allows rapid design and production of MIPs inexpensively; ready to be tested in a very short time. Recently, we have shown that nanoMIP-based optical sensor could be developed for endotoxin monitoring. This was achieved by using itaconic acid as the best monomer for MIP 
synthesis and the affinity-based endotoxin assay could detect the endotoxins in the concentration range of $15.6-500 \mathrm{ng} \mathrm{mL}^{-1}$ with dissociation constants of $3.24-5.24 \times 10^{-8} \mathrm{M}$ [19]. The surface plasmon resonance (SPR) is a highly sensitive label free optical sensor. The MIPs produced can be immobilized onto a gold SPR sensor coated in a self-assembled monolayer (SAM) through EDC/NHS coupling of the carboxyl on the sensor and primary amine groups on the MIPs [20]. The interaction between the MIP and the analyte can then be measured to determine if the MIP can successfully detect and capture the target. In this study the endotoxin template was used to design and synthesis tailor-made nanoMIPs for the endotoxin. The nanoMIPs were characterized to determine the quality, reproducibility and properties of each MIP produced with the functional monomers itaconic acid (IA), methacrylic acid (MA) and acrylamide (AA). The three alternative endotoxin MIPs with different binding energies provided parallel results with computational simulation in the sensor; the MIP with highest binding energy gave the highest signal on the sensor. A novel method for the preparation of endotoxin solutions with triethylamine resulted in endotoxin subunits, which were then conjugated to gold nanoparticles efficiently without agglomeration. This enhanced the detection signal on the SPR biosensor platform, and showed ultrasensitive detection of endotoxins and improvement in the affinity of MIPs against the endotoxin target. The experimental design of entire research work from computational design of MIP receptors to affinity based AuNP functionalized endotoxin assays on the MIP immobilized sensor was given in Scheme 1.

\section{Materials and Methods}

\subsection{Reagents and chemicals}


Endotoxins from Escherichia coli 0111:B4 purified by ion exchange chromatography, 1ethyl-3-(3-dimethylaminopropyl) carbodiimide (EDC), N-hydroxysuccinimide (NHS) 98\%, phosphate buffered saline (PBS), ethanolamine hydrochloride, vancomycin hydrochloride, HEPES buffer, glutaraldehyde (GA), 3- aminopropyltrimethyloxysilane (APTMS), 11mercaptoundecanoic acid (MUDA) 95\%, ethanol, $60 \mathrm{~mL}$ SPE tubes and $20 \mu \mathrm{m}$ pore frits, Acetonitrile (ACN), itaconic acid (IA), pentaerythritol tetrakis(3-mercaptopropionate) 95\% (PETMP), trimethylolpropane trimethacrylate (TRIM), ethylene glycol dimethacrylate (EDGMA), N,N-Dimethylformamide (DMF), methacrylic acid (MA), acrylamide (AA), triethylamine and chloroform were purchased from Sigma Aldrich (UK). Benzyl diethyldithiocarbamate (iniferter) was from TCI Chemicals (EU). Toluene, acetone and sodium hydroxide were purchased from Fisher Scientific (UK). N-(3-aminopropyl) methacrylamide hydrochloride was purchased from Polyscience Inc (Germany). Glass fibre syringe filters 0.45 $\mu \mathrm{m}$ were from Jaytee Biosciences Ltd. (Kent, UK). Double- distilled ultrapure water produced by a Millipore Direct-Q ${ }^{\circledR} 3$ UV (Millipore; Molsheim, France) was used for analysis. Glass beads (Spheriglass ${ }^{\circledR}$ 2429, $53 \mu \mathrm{m}<$ diameter $<106 \mu \mathrm{m}$ ) were from Blagden Chemicals (UK). All chemicals and solvents were analytical or HPLC grade and were used without further purification.

\subsection{Apparatus and equipment}

Ceramic beads with glass beads were agitated by a Retsch AS200 shaker (Retsch Inc.). A Zetasizer Nano (Nano-S) from Malvern Instruments Ltd. (Malvern, U.K.) was employed to determine the size of the nanoparticles by dynamic light scattering (DLS). SPR-4 sensor with its gold-coated sensor chips was supplied by Sierra Sensors (Germany). A transmission electron 
microscope (TEM) (Philips CM20, Philips Research) and a scanning electron microscope (SEM) (JEOL-JSM5800LV) were employed to characterize the MIPs nanoparticles.

\subsection{Computer modeling for MIP design}

The endotoxin structure as the template from Escherichia coli 0111:B4 was acquired from the human metabolome database (HMDB) and its 2D structure was transformed to $3 \mathrm{D}$ for minimization purposes. The molecule was then charged by the Gasteriger-Huckel method and the molecular mechanics was applied to minimize the structure using the Powell method. The minimization was run for 10,000 iterations or until the convergence gradient reached $0.001 \mathrm{kal}$ $\mathrm{mol}^{-1}$. The template was screened with the 21 most common monomers used in MIP synthesis. The results were ranked from highest to lowest according to the binding energy obtained by the LEAPFROG $^{\mathrm{TM}}$ algorithm. The selection was further narrowed down using the visualization tool to only select monomers that were interacting with the conserved region of the endotoxin. The full procedure is outlined in elsewhere $[15,19]$.

\subsection{Synthesis of MIP nanoparticles}

A detailed explanation of the procedure is highlight in previous works [19, 21]. Glass beads with a diameter of $75 \mu \mathrm{m}$ were silanized with a short linker APTMS to allow the endotoxin template to be immobilized using glutaraldehyde (Scheme 2). The polymerization mixture for endotoxin MIPs was adapted from [16]. Three alternative endotoxin MIPs designed based on molecular modelling results were produced using itaconic acid (2.18 g), methacrylic acid (1.44 g) or acrylamide (1.19 g) as functional monomers. Trimethylolpropane trimethacrylate (TRIM) and ethylene glycol dimethacrylate (EDGMA) were used as cross-linkers in the amount of 1.62 g. The ratio between each functional monomer and cross-linkers was 1.29:1 as mol. DMF was 
used to dissolve the polymer mixture of IA-MIP whereas ACN was added to MA-MIP and AAMIP compositions. As having too low computational score with very low molecular affinity, styrene was also used as a low score monomer to imprint endotoxin. N-(3-aminopropyl) methacrylamide was used as additional monomer to functionalize primary amine groups to the surface of the MIPs nanoparticles by exposing to UV for 30 seconds. Addition of amine group to the MIPs is critical since they need to be attached to MUDA coated sensor surface via covalent interaction during endotoxin detection assays. High affinity endotoxin nanoMIPs were obtained after three step polymerization [19]. Vancomycin MIP nanoparticles were produced as control MIPs using the same method with identical polymer composition and characterized.

\subsection{Characterization of MIPs nanoparticles}

The synthesized MIP nanoparticles were characterized by DLS to verify the size and quality of the production. The yield of each batch was also determined by evaporation. Since the synthesized MIPs were obtained in a solution, this solution was evaporated to leave the MIP in a dry format which was then weighed to determine the yield. A transmission electron microscope (TEM) was used for TEM images of the synthesized MIP nanoparticles. Concentrated solutions of MIPs were used by filtering $5 \mu \mathrm{l}$ sample through a $1.2 \mu \mathrm{m}$ glass fibre syringe filter and deposited on a silicon chip attached to a TEM holder, and left to dry overnight in a fume hood. A scanning electron microscope (SEM) image of the MIPs nanoparticles were also taken by scanning electron microscopy.

\subsection{NanoMIP-based endotoxin sensor assays}

Stock solutions of endotoxins were prepared in $1 \mathrm{~mL}$ HEPES buffer (10 mM, pH 7.0) with equimolar triethylamine. The sample was heated to $60{ }^{\circ} \mathrm{C}$ for 5 minutes and then vortexed 
until a homogenous solution of endotoxins was obtained. Endotoxins were then functionalized with gold nanomaterials using a well-established method [16] to amplify SPR signal for the detection of trace amounts. All endotoxin concentrations tested were verified using the limulus amebocyte lysate (LAL) assay and the molecular weight for endotoxin used was 20,000 Da.

The affinity between the endotoxin and the synthesized MIPs was investigated using an SPR sensor. The running buffer and endotoxin analyte samples were prepared using HEPES buffer (10 mM, pH 7.0). The MIP nanoparticles were concentrated by evaporation to determine concentration by weight and then resuspended in $1 \mathrm{~mL}$ of MES buffer (10 mM, pH 6.0) before investigating the best immobilization conditions. The MUDA coated SPR-4 sensor chip was activated using $100 \mu 1$ of EDC/NHS solution [20] prior to the covalent immobilization of MIP nanoparticles $\left(600 \mu \mathrm{g} \mathrm{mL} \mathrm{m}^{-1}\right)$ on the sensor spots. SPR-4 system has four separate sensing channels. Therefore, three alternative endotoxin MIPs were simultaneously immobilized and the fourth channel was used for the control MIP. A $100 \mu$ l ethanolamine (1 M) was added to block MIP free areas on the surfaces to prevent from non-specific binding during endotoxin detection assays. Endotoxin samples were injected (100 $\mu$ l per sample) sequentially from lower to higher concentrations in the range of $0.2-200 \mathrm{ng} \mathrm{mL}^{-1}$. Kinetic data has been analyzed and manipulated using SPR-4 software analyzer (Sierra Sensors, Germany). The dissociation constants were calculated from experimental data using the Sierra Sensors SPR analyser software. The parameters were set within the software to highlight the start and end of an analyte cycle and then the software compares the control results to calculate the $K_{D}$ values based on different analyte concentrations.

\section{Results and Discussions}




\subsection{Computational design of nanoMIPs}

The molecular modeling results obtained using the LEAPFROG $^{\mathrm{TM}}$ algorithm are as shown in Table 1. The functional monomers were selected according to their binding score and were reduced to just three monomers using the visualization technique. This technique aided in selection of monomers that bind to the conserved region of the endotoxin structure and monomers that competed for the same site were removed according to which had the highest score. There were instances of functional monomers with high binding scores interacting to areas of the endotoxin with no significance. These monomers were eliminated from the selection, as they would have produced non-specific MIPs [22]. This clearly demonstrates the cost effective approach of molecular modeling as it has already significantly reduced the number of unnecessary experiments that saves time and materials.

It is not possible to create non-imprinted polymers as controls using the solid phase technique, as material will not be retained in the absence of a template [23]. Therefore, vancomycin MIPs was used as controls as the functional monomers also displayed similar results towards the vancomycin template that are comparable to the endotoxin template (Table 1). Itaconic acid (IA) and acrylamide (AA) had very similar scores for both endotoxin and vancomycin whereas methacrylic acid (MA) showed a clear difference of $14.14 \mathrm{Kcal} \mathrm{mol}^{-1}$. The vancomycin MIPs has identical polymer compositions and the size of the MIPs is slightly larger which act as suitable controls to determine the selectivity of the MIPs towards endotoxin. Fig. 1 displays the visualization of the monomers IA and MA forming hydrogen bonds with a phosphate group in the conserved region of the endotoxin structure, while AA displays hydrogen bonds targeting two phosphate groups. The monomers show the capability of assembling around these key groups and by cross linking the functional monomers, MIPs produced should bind to 
the endotoxin selectively. In addition to the best possible monomer candidates coming from computational modelling, styrene based endotoxin-MIP was also imprinted as having a very low binding energy $\left(-8.47 \mathrm{Kcal} \mathrm{mol}^{-1}\right)$ to see the efficiency of the modelling approach in real application.

\subsection{Solid-phase MIP nanoparticle synthesis}

The MIPs for endotoxin and vancomycin were synthesized using the solid-phase approach with UV photopolymerization. MIPs produced for endotoxin and vancomycin all had identical polymer composition. Three alternative MIPs were produced using three different monomers (IA, MA, AA) based on the computational simulation and all were then characterized using DLS, SEM and TEM techniques and tested for affinity using the SPR sensor. DLS characterization of the MIPs were performed after they were sonicated for 30 seconds to reduce aggregation before filtering using a glass fibre syringe $(0.45 \mu \mathrm{m})$ for the removal of dust and large particles. Three batches of endotoxin MIPs were prepared for each monomer and overall results are displayed in Table 2. MIPs produced with the functional monomers IA and MA averaged $203 \pm 13 \mathrm{~nm}$ and $219 \pm 14 \mathrm{~nm}$ in diameter, respectively. The PDI results indicated the polymers were monodisperse. This highlights the quality and reliability of the process used to synthesis the MIPs. MIPs produced with AA as the functional monomer averaged $235 \pm 34 \mathrm{~nm}$ in diameter with the PDI of $0.389 \pm 0.03$. The size variation of different endotoxin MIPs can be related to closer interaction of highest affinity monomer with endotoxin resulting in smaller size MIPs as this was observed from computational simulations (the highest affinity monomer (IA) provided smallest size MIP; and MA and AA followed this gradually. Moreover, the standard deviation of size was smaller in the case of higher affinity monomers which may highlight less flexible MIP structure. The vancomycin MIPs as the control were also characterized and the size 
of control nanoMIPs were found in the range of 240-270 nm with PDI of 0.3-0.4. All the results for three alternative endotoxin MIPs and control MIPs were reported (Table 2). The yield of each MIP production was also determined by evaporation. The maximum yield was obtained with IA based endotoxin MIP (14.7 mg) and this was followed by MA-MIP (11.5 mg) and AA-MIPs (10.6 mg). Low affinity endotoxin MIP imprinted with styrene as functional monomer gave a PDI higher than 0.6 which indicates unsuccessful imprinting with very high polydispersity and polymer fragments instead of obtaining a monodisperse, stabile and high quality MIP. The size of styrene based endotoxin MIP also showed a big variation (100-300 nm) and confirm the significance of the computational modelling results

Further characterization of the synthesized MIP nanoparticles was performed by TEM and SEM analysis (Fig. 2) which shows a single uniform particle resulting from the solid phase synthesis procedure used in this work.

\subsection{Ultrasensitive endotoxin sensor assay}

The surface plasmon resonance optical biosensor was successfully employed to determine the selectivity and affinity of the synthesized endotoxin MIPs for comparative studies. SPR analysis was used to identify the lowest detection limit for the endotoxins to determine the sensing capabilities and its ability to retain the target for detection and removal purposes. Three alternative endotoxin MIPs, which were designed based on computational simulations, were investigated. Comparative analysis of MIPs for endotoxin detection by SPR sensor gave similar results with computational modelling. As being the best monomer with the highest binding score, IA produced higher signal in the sensor assays. A clear response difference was also recorded between second (MA) and third (AA) best monomer (Fig. 3A). Moreover, free endotoxin 
samples (without gold nanoparticle functionalization) were tested with same concentration of endotoxin which could not produce any measurable binding response when compared to the baseline. An endotoxin concentration of $25 \mathrm{ng} \mathrm{mL}^{-1}$ was selected to clearly highlight the binding differences between the three endotoxin MIPs to be compared with the control assay. The MIPs containing the IA have the highest binding responses of $99.5 \mathrm{RU}$ followed by MA containing MIPs 66.4 RU while MIP containing AA obtained a response of 35.6 RU (Fig. 3A). The SPR sensorgram also show very little dissociation for the endotoxin samples in the concentration range of 1-200 $\mathrm{ng} \mathrm{mL}^{-1}$. This is promising for the capture and removal of endotoxin from an aqueous environment as the MIPs are capable of binding to its target.

The vancomycin MIP controls did not display significant binding to the endotoxin despite identical polymer composition (Fig. 3B). The control MIP containing IA shows a non-specific binding of $16 \mathrm{RU}$ for $25 \mathrm{ng} \mathrm{mL}^{-1}$ endotoxin concentration, which is almost a fifth of what is achieved by the endotoxin MIP containing IA. The MIPs produced using the functional monomers MA and AA show very low response ( 1-2 RU). The success of endotoxin detection in terms of limit of detection and $K_{D}$ has significantly improved in this work compared to our previous work [19] due to the new method developed for endotoxin sample preparation with triethylamine.

The limit of detection was calculated for each endotoxin MIP: IA was capable of detecting $0.438 \pm 0.023 \mathrm{ng} \mathrm{mL}^{-1}$, MA $0.828 \pm 0.044 \mathrm{ng} \mathrm{mL}^{-1}$ and AA $1.622 \pm 0.087 \mathrm{ng} \mathrm{mL}^{-1}$ (Fig. 4). The improvements were largely due to the preparation of the endotoxins with equimolar triethylamine as this would disaggregate the endotoxin into subunits and allow functionalization of the gold nanoparticles more effectively [24]. A $\sim 40$ times lower detection limit was achieved in this work with the new strategy which has a fundamental role to detect trace amounts of 
endotoxins. The chart in Figure 4 is modeled on one site binding and the $\mathrm{R}^{2}$ values were found to be $0.983,0.993$ and 0.964 for IA, MA and AA respectively providing a high level of confidence in the data.

The dissociation constants were also calculated using the Langmuir binding model to determine the mathematical affinity between each endotoxin MIP and endotoxin. The $\mathrm{K}_{\mathrm{D}}$ was calculated as $5.24 \times 10^{-10} \mathrm{M}, 4.4 \times 10^{-10} \mathrm{M}$ and $1.63 \times 10^{-8} \mathrm{M}$ for IA-, MA- and AA-MIPs respectively (Table 3). With the new method, the affinity was successfully increased from $3.24 \mathrm{x}$ $10^{-8} \mathrm{M}$ to $5.24 \times 10^{-10} \mathrm{M}$ for IA-MIP as reported in our previous work. The aggregation would have prevented the MIPs from binding to the conserved regions effectively. The endotoxins in a subunit formation allow consistent binding to occur for more accurate readings to be obtained. Vancomycin MIPs produced with IA had a $K_{D}$ value of $6.52 \times 10^{-6} \mathrm{M}$, which is significantly lower than what was determined for endotoxin MIPs. Dissociation values for vancomycin MIPs containing MA and AA could not be calculated as responses were too low.

The MIPs clearly display a high affinity and sensitivity towards the endotoxins and the MIP surface could be completely regenerated by applying $20 \mathrm{mM} \mathrm{NaOH}$. This demonstrates the ability of multiple uses (<30 times) of MIPs highlighting the stability of these polymers (Fig. 5). IA-MIP and MA-MIP have shown very consistent results during 30 regeneration, whereas AAMIP lost its regeneration ability up to $70 \%$ after cycle 12 . This is also a confirmation of molecular modelling results which provided lowest binding score for AA-MIP among three alternative MIPs. Regeneration ability of MIPs is important feature to detect low concentration of a target several times without losing sensitivity and to efficiently retain the analyte to be considered an effective affinity ligand towards the endotoxin. Batch-to-batch variation of AuNP functionalized endotoxins was also evaluated in this work since it is vital to obtain a consistent 
conjugation efficiency. For this, two different batches were compared and the deviation on the data was found less than $10 \%$ which highlights the success of the method developed with triethylamine (Fig. 6) The use of triethylamine is an important addition to the endotoxin sample as the formation of smaller aggregates or monomer units is important for endotoxin bioactivity and the bioactivity is directly linked to the lipid A region of the endotoxin. The method provides a significant consistency on the conjugation while allowing the MIP to bind to the bioactive region. This cannot be achieved without the use of triethylamine because the endotoxin molecule tends to form large micelle structures due to its amphipathic characteristic that causes interference with gold nanoparticle solution; hence, also cause low affinity during the binding assays.

The gold standard affinity ligand Polymyxin B for the endotoxin has a $K_{D} 1.7 \times 10^{-7} \mathrm{M}$ on the SPR determined by Thomas and Surolia [25]. As being the other alternative affinity ligand, aptamers have also been applied in biosensor-based endotoxin detection. A detection limit of $0.1 \mu \mathrm{g} \mathrm{mL} L^{-1}$ was achieved with $\mathrm{K}_{\mathrm{D}}$ in $\mathrm{nM}$ range by employing a SPR sensor [26] whereas impedance-based electrochemical biosensor has provided LPS detection in the range of 0.001-1 ng $\mathrm{mL}^{-1}$ with similar affinity [27]. However, the designing and producing aptamers are much more difficult than nanoMIPs and requires significantly longer time. Design and synthesis of three endotoxin specific MIPs requires only two months in our work whereas it takes more than six months to develop one specific aptamer. Moreover, one batch of production has resulted in very high amount with our methodology to be used for long term testing of endotoxin.

The use of natural antibodies for endotoxin detection by employing biosensors is very limited and recent developments are mainly relying on Polymyxin B, aptamers and endotoxinneutralizing protein [28]. This can be due to the difficulties arising from agglomeration of 
endotoxin during the bioassays as well as lack of the required specificity and affinity of the antibodies. In the current work, agglomeration problem was successfully solved using our novel trimethylamine method which can also be coupled with other affinity ligand based endotoxin detection; thus, enable the extensive usage of natural antibodies and biosensors in the area. In comparison the $\mathrm{K}_{\mathrm{D}}$ for the endotoxin MIPs $\left(5.24 \times 10^{-10} \mathrm{M}\right)$ display an improved performance according to the kinetic data analysis. The endotoxin detection techniques with biosensors have been recently reviewed [13] and our recent work has highlighted the superiority over these investigations.

\section{Conclusions}

Selective molecularly imprinted polymers for endotoxins were designed, synthesized and tested. The computational approach has demonstrated its potential in the design of MIPs displaying selectivity and affinity towards the endotoxin. These MIPs were significantly higher in affinity and specificity compare to all of the vancomycin MIP controls containing IA, MA and AA. This approach to produce endotoxin MIPs is cost effective eliminating unnecessary experiments reducing the use of organic solvents and expensive materials such as the template endotoxin. Three alternative endotoxin MIPs synthesized by employing IA, MA and AA as functional monomers resulted in the yield of $14.6 \mathrm{mg}, 11.5 \mathrm{mg}$ and $10.6 \mathrm{mg}$. The best monomer (IA) provided larger amount of MIPs for each batch and the other two monomers also gave correlative yield with respect to computational simulation scores. Moreover, this similarity was also found in SPR results where the IA-MIP produced the highest signal with the best detection limit and the results for the other two endotoxin MIPs followed this consecutively. Significant progress has been achieved in increasing the sensitivity of endotoxin detection and $\mathrm{K}_{\mathrm{D}}$. Detection limit has been improved from [19] $15.6 \mathrm{ng} \mathrm{mL}^{-1}$ to $0.44 \mathrm{ng} \mathrm{mL}^{-1}$ (current work) and 
the $\mathrm{K}_{\mathrm{D}}$ from $3.24 \times 10^{-8} \mathrm{M}$ to $4.4 \times 10^{-10} \mathrm{M}$ with the aid of new sample preparation technique which was developed using triethylamine. Furthermore these MIPs for the endotoxin can be regenerated and reused more than 30 times without significant decrease in binding response allowing the use of one sensor several times. The current work has demonstrated the potential of the technology for ultrasensitive endotoxins detection and risk management and also the importance of computational modeling to design the artificial affinity ligands.

\section{Author Contributions}

$\dagger$ Z.A. and M.J.A. equally contributed to this work

\section{Notes}

The authors declare no competing financial interest.

\section{Acknowledgment}

We gratefully acknowledge the European Commission for supporting this work (FP7-280595) "NANOPUR project".

\section{References}

[1] U. Ohto, K. Fukase, K. Miyake, T. Shimizu, Proc Natl Acad Sci, 109 (2012) 7421-7426.

[2] A.P. Zavascki, L.Z. Goldani, J. Li, R.L. Nation, J. Antimicrob. Chemother 60 (2007) 12061215.

[3] J. Andra, K. Lohner, S. Blondelle, R. Jerala, I. Moriyon, M. Koch, P. Garidel, K. Brandenburg, Biochem.J. 385 (2005) 385, 135-143.

[4] J.C. Hurley, Toxins 5 (2013) 2589-2620. 
[5] C. Tischer, U. Gehring, C. M. Chen, M. Kerkhof, G. Koppelman, S. Sausenthaler, O. Herbarth, B. Schaaf, I. Lehmann, U. Kramer, D Berdel, A. von Berg, C.P. Bauer, S. Koletzko, H.E. Wichmann, B. Brunekreef, J. Heinrich, Eur Respir J. 37 (2011) 1050-1059.

[6] Z. Can, L. Wenjun, S. Wen, Z. Minglu, Q. Lingjia, L. Cuiping, T. Fang, Water Res. 47 (2013) 3591-3599.

[7] K. Ogawa, M. Hyuga, T. Okada, N. Minoura, Biosens. Bioelectron. 38 (2012) 215-219.

[8] S. Leone, A. Silipo, E.L. Nazarenko, R. Lanzetta, M. Parrilli, A. Molinaro, Mar. drugs 5 (2007) 85-112.

[9] S. Copeland, H.S. Warren, S.F. Lowry, S. E. Calvano, D. Remick, Clin Vaccine Immunol $12(2005) 60-67$.

[10] D. Landman, C. Georgescu, D.A. Martin, J. Quale, Clin. Microbiol. Rev. 21 (2008) 449465.

[11] H. Tsubery, H. Yaakov, S. Cohen, T. Giterman, A. Matityahou, M. Fridkin, I. Ofek, Antimicrob. Agents. 49 (2005) 3122-3128.

[12] H. Tsubery, I. Ofek, S. Cohen, M. Eisenstein, M. Fridkin, Mol. Pharmacol. 62 (2002) 1036.

[13] A. Das, P. Kumar, S. Swain, Biosens. Bioelectron. 51 (2014) 62-75.

[14] K. Karim, F. Breton, R. Rouillon, E.V. Piletska, A. Guerreiro, I. Chianella, S.A. Piletsky, Adv. Drug Deliv. Rev. 57 (2005) 1795-1808.

[15] I. Chianella, K. Karim, E.V. Piletska, C. Preston, S.A. Piletsky, Anal. Chim. Acta 559 (2006) 73-78.

[16] Z. Altintas, A. Guerreiro, S.A. Piletsky, I.E. Tothill, Sens. Actuators, B. 213 (2015) 305313. 
[17] Z. Altintas, M. Gittens, A. Guerreiro, K.A. Thompson, J. Walker, S. Piletsky, I.E. Tothill, Anal. Chem. 87 (2015) 6801-6807.

[18] A.R. Guerreiro, I. Chianella, E. Piletska, M.J. Whitcombe, S.A. Piletsky, Biosens. Bioelectron. 24 (2009) 2740-2743.

[19] M.J. Abdin, Z. Altintas, I.E. Tothill, Biosens. Bioelectron. 67 (2015) 177-183.

[20] Z. Altintas, Y. Uludag, Y. Gurbuz, I.E. Tothill, Anal. Chim. Acta 712 (2012) 138-144.

[21] E. Moczko, A. Poma, A. Guerreiro, I.P. Vargas Sansalvador, S. Caygill, F. Canfarotta, M.J. Whitcombe, S. Piletsky, Nanoscale 5 (2013) 3733-3741.

[22] Z. Altintas, B. France, O.J. Ortiz, I.E. Tothill, Sens. Actuators, B. 224 (2016) 726-737.

[23] A. Poma, A. Guerreiro, M.J. Whitcombe, E.V. Piletska, A.P. Turner, S.A. Piletsky, Adv Funct Mater. 23 (2013) 2821-2827.

[24] I. Chafchaouni-Moussaoui, A. Novikov, F. Bhrada, M.B. Perry, A. Filali-Maltouf, M. Caroff, Rapid Commun Mass Spectrom. 25 (2011) 2043-2048.

[25] C.J. Thomas, A. Surolia, FEBS Lett. 445 (1999) 420-424.

[26] M.S.A. Rahman, S.C. Mukhopadhyay, P.-L. Yu, J. Goicoechea, I.R. Matias, C.P. Gooneratne, J. Kosel, Food Eng. 114 (2013) 346-360.

[27] W. Su, M. Lin, H. Lee, M. Cho, W.-S. Choe, Y. Lee, Biosens. Bioelectron. 32 (2012) 3236.

[28] W. Limbut, M. Hedström, P. Thavarungkul, P. Kanatharana, B. Mattiasson, Anal. Bioanal. Chem. 389 (2007) 517-525. 


\section{Legends of Figures}

Scheme 1. Experimental design of entire research work from computational design of MIP receptors to affinity based AuNP functionalized endotoxin assays on the nanoMIP immobilized sensor.

Scheme 2. Schematic illustration of glass bead preparation with template for solid-phase MIP synthesis.

Fig. 1. Stoichiometric complex visualization of functional monomers interacting with the 3D structure of the endotoxin template resulted from the computer simulations, forming hydrogen bonds with the conserved region of the endotoxins that contain phosphate groups. Different colors represents different atom/bond. Light blue: hydrogen, white: carbon, dark blue: nitrogen, red: oxygen.

Fig. 2. SEM (A) and TEM (B) images of endotoxin nanoMIPs. Inner parts show single MIP particle under SEM and TEM.

Fig. 3. Real-time affinity based sensor assays using SPR-4 biosensor platform. The endotoxin detection sensorgram, comparing the binding affinity between endotoxin nanoMIP containing IA, MA and AA with endotoxin conjugated to gold (A). Control assays using vancomycin MIPs. Real-time sensorgram displays the binding interaction of AuNP conjugated endotoxin (25 ng $\mathrm{mL}^{-1}$ ) on the control MIPs (B).

Fig. 4. One site binding curves for overall endotoxin detection assays with three alternative endotoxin MIPs in the concentration range of $1.56-200 \mathrm{ng} \mathrm{mL}^{-1}$. Each endotoxin sample was functionalized with gold nanoparticles using new triethylamine method 
Fig. 5. Regeneration ability of endotoxin MIPs on the sensor surface during 30 cycles of endotoxin binding.

Fig.6. Batch-to-batch variation of AuNP functionalized endotoxin assays using triethylamine method. 


\section{Legends of Tables}

Table 1. Functional monomers selected through the computational modelling and screening process for the endotoxin and vancomycin MIP ranked from highest to lowest energy ranking.

Table 2. The mean and standard deviation of the diameter and polydispersity (PDI) readings obtained using DLS for each endotoxin (Endo) and vancomycin (Vanc) MIP synthesis.

Table 3. Affinity analysis of the interaction between endotoxin and vancomycin MIPs with gold nanomaterials conjugated endotoxin, calculated as dissociation constant $\left(\mathrm{K}_{\mathrm{D}}\right)$. 


\section{List of Figures}

\section{Scheme 1}

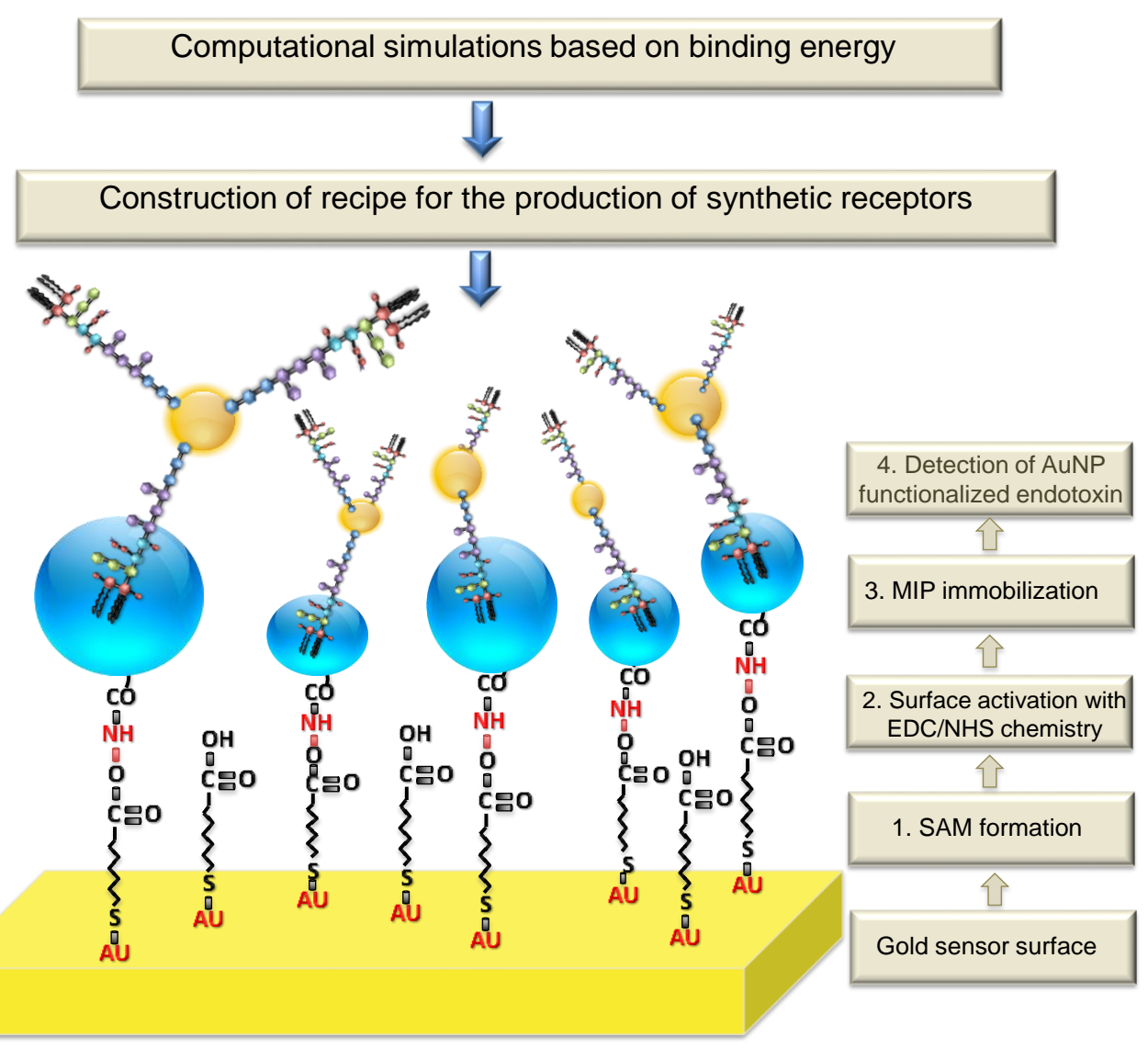


Scheme 2

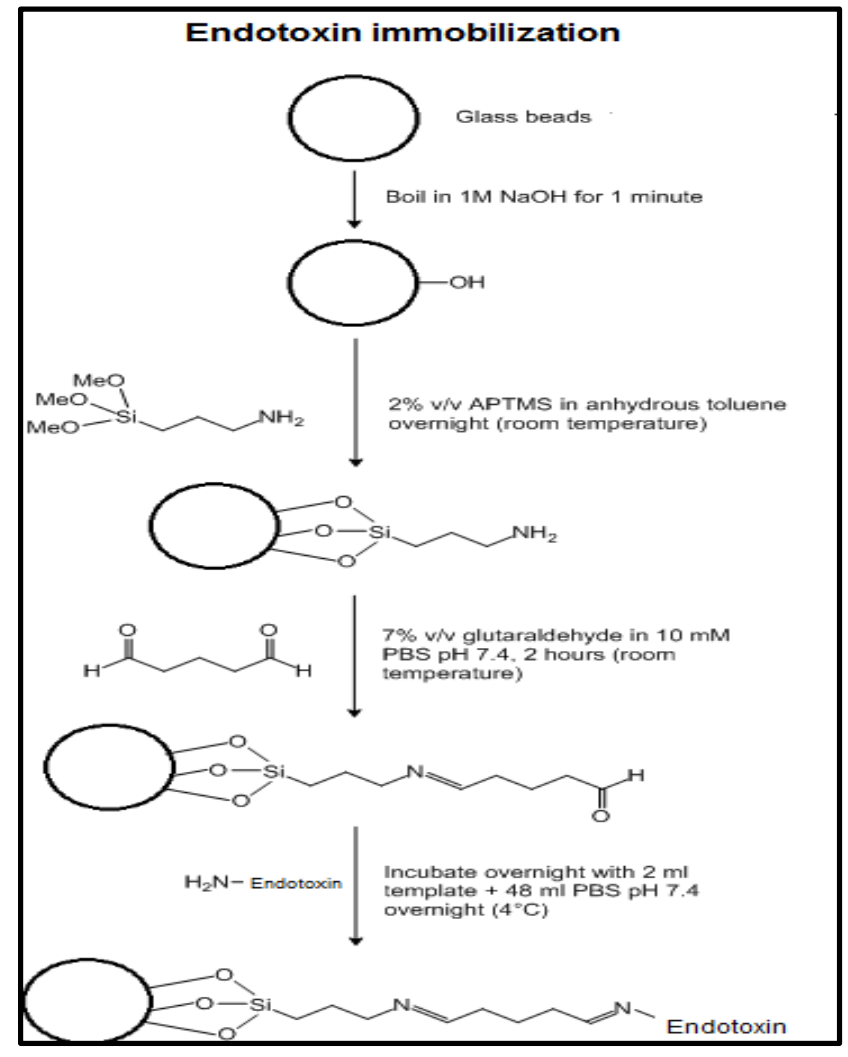




\section{Figure 1}

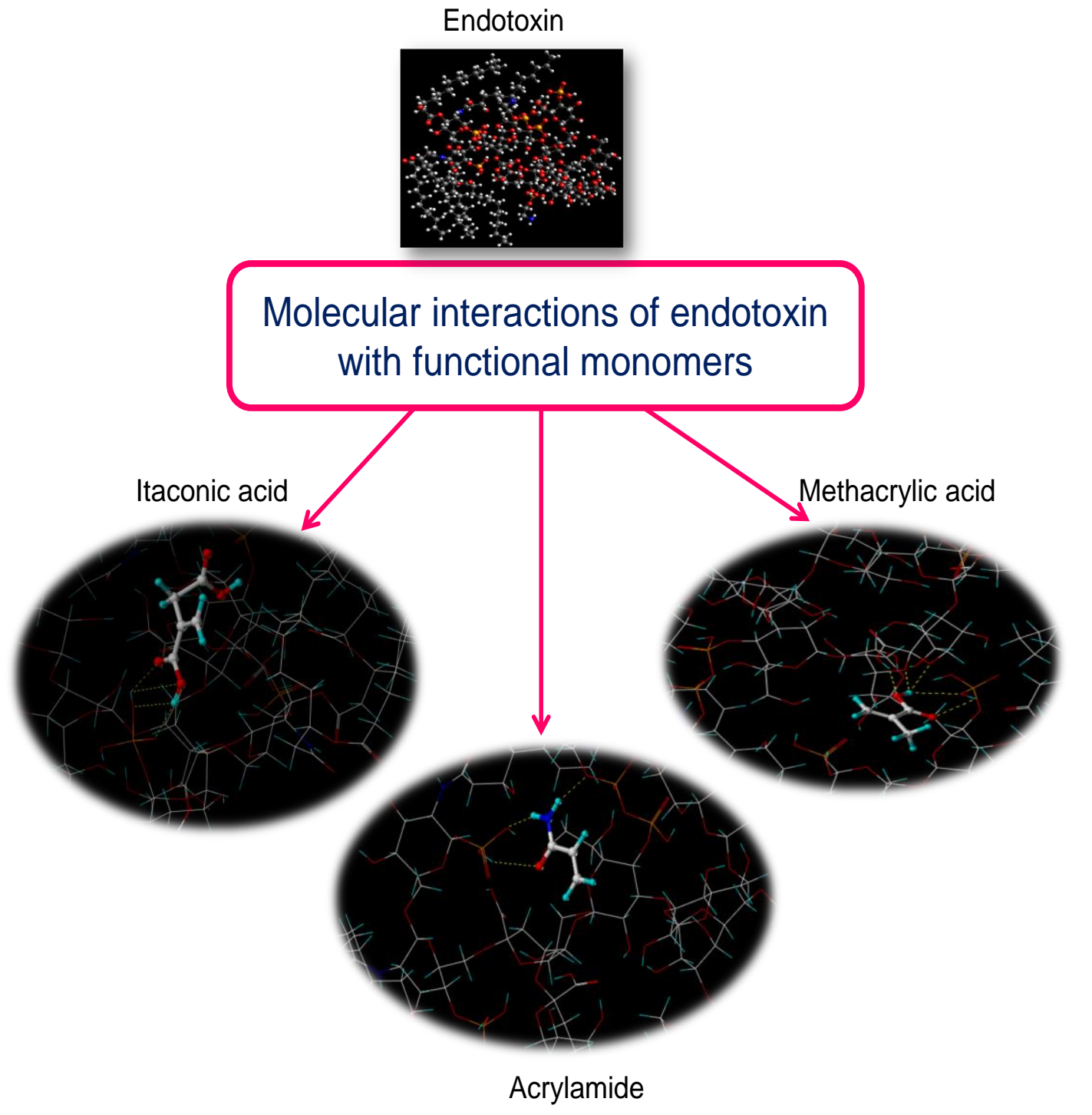


Figure 2
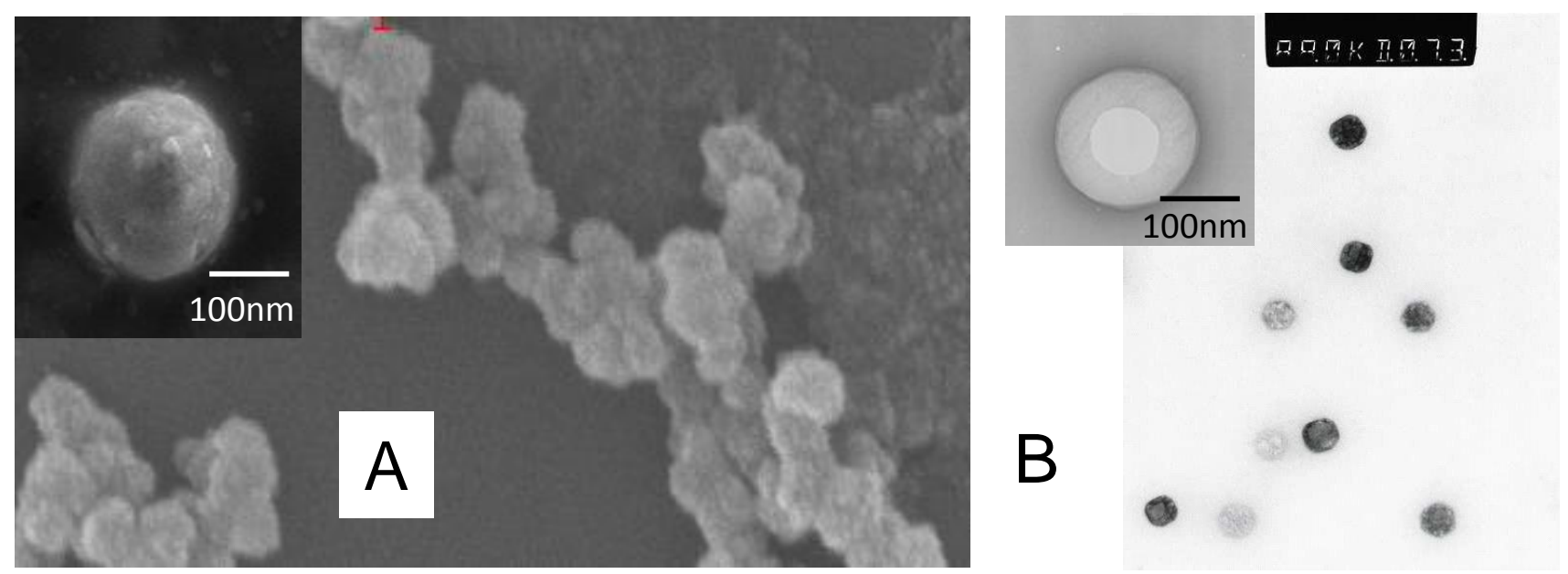


\section{Figure 3}
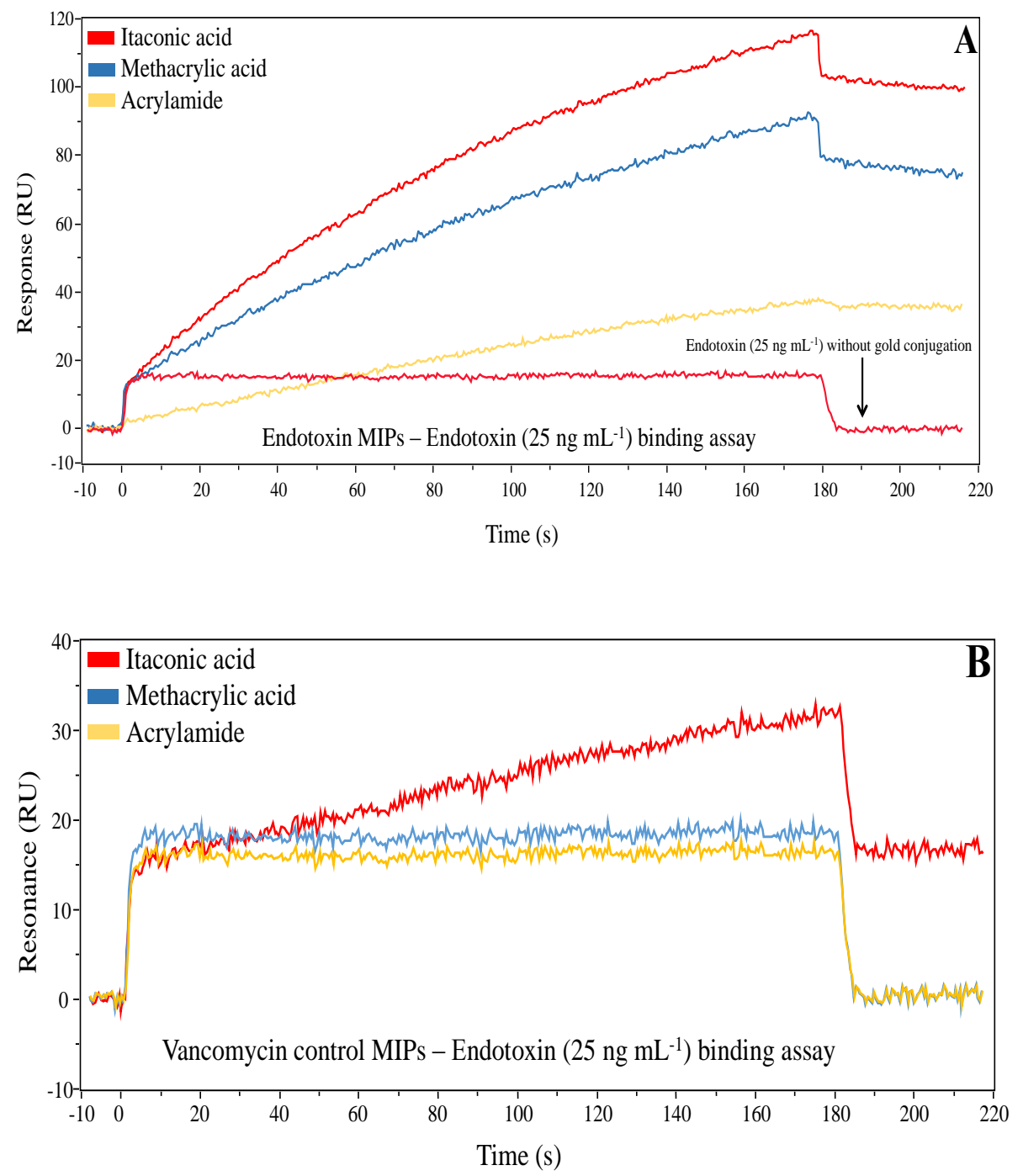


\section{Figure 4}

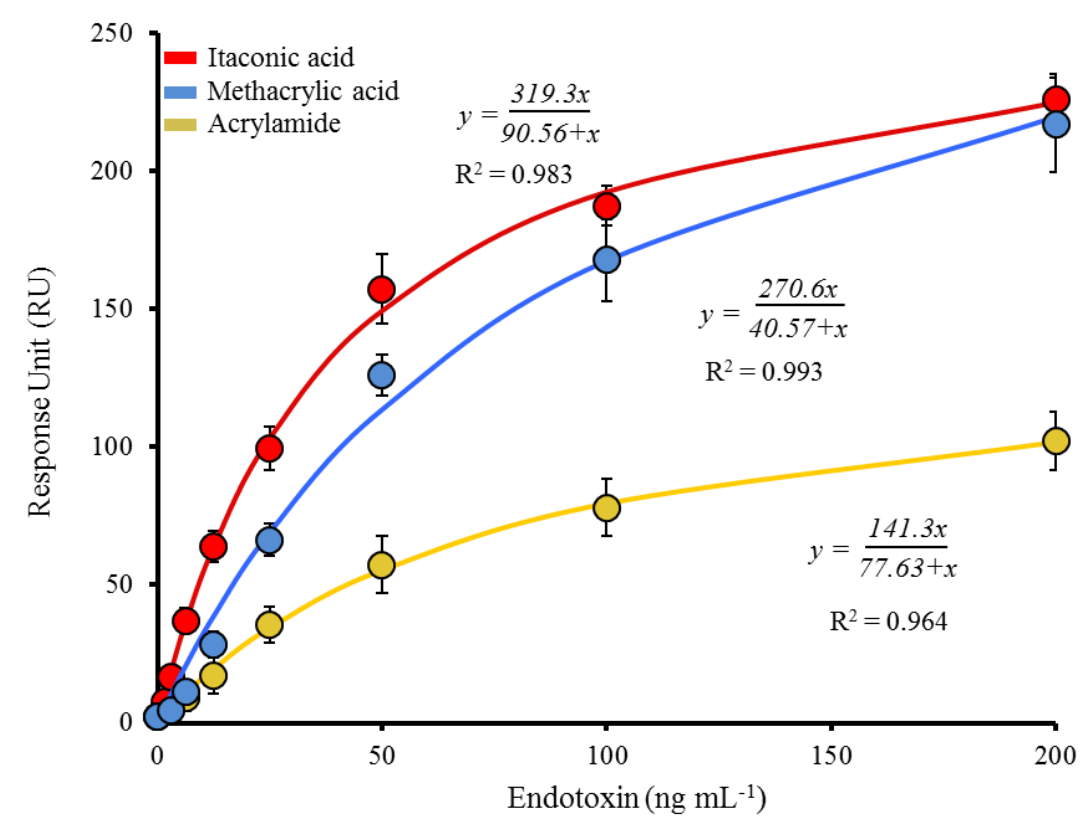




\section{Figure 5}

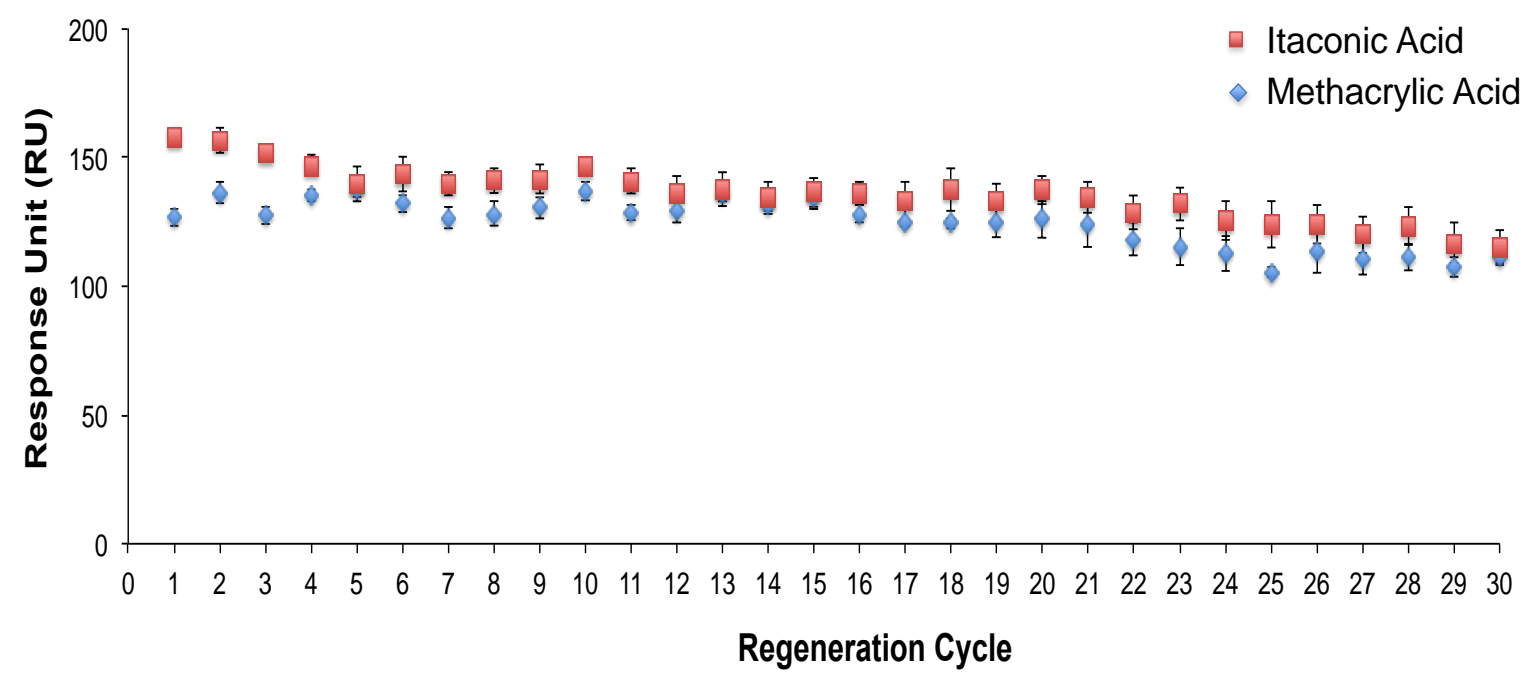




\section{Figure 6}

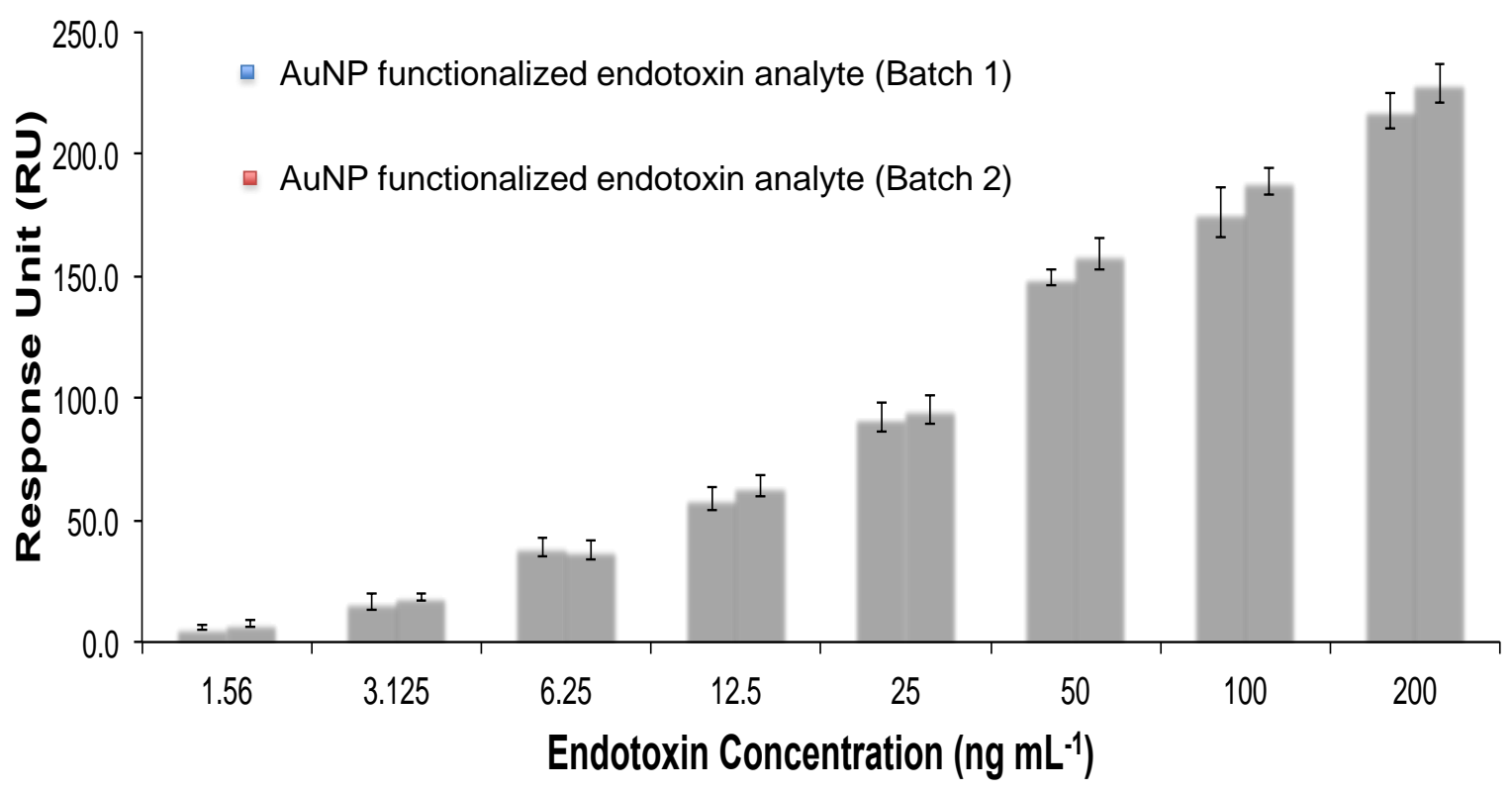




\section{List of Tables}

\section{Table 1}

\begin{tabular}{|llll|}
\hline Structure & Monomer & Binding to endotoxin & Binding to vancomycin \\
& & $\left(\mathrm{Kcal} \mathrm{mol}^{-1}\right)$ & $\left(\mathrm{Kcal} \mathrm{mol}^{-1}\right)$ \\
\hline & & & -48.76 \\
& Methacrylic acid & -41.43 & -27.29 \\
\hline & & & -36.68 \\
\hline
\end{tabular}


Table 2

\begin{tabular}{|lll|}
\hline & Diameter [nm] & PDI \\
\hline Endo MIPs IA & $202.8 \pm 13$ & $0.251 \pm 0.02$ \\
Endo MIPs MA & $219.0 \pm 14$ & $0.307 \pm 0.05$ \\
Endo MIPs AA & $235.0 \pm 34$ & $0.389 \pm 0.03$ \\
Vanc MIPs IA & $263.5 \pm 28$ & $0.320 \pm 0.04$ \\
Vanc MIPs MA & $272.5 \pm 10$ & $0.362 \pm 0.07$ \\
Vanc MIPs AA & $241.5 \pm 10$ & $0.424 \pm 0.04$ \\
\hline
\end{tabular}

*Itaconic Acid (IA), Methacrylic Acid (MA), Acrylamide (AA). 


\section{Table 3}

\begin{tabular}{|lllll|}
\hline MIPs & Binding model & Dissociation & Yield $(\mathrm{mg})$ & $\begin{array}{c}\text { Limit of detection } \\
\left(\mathrm{ng} \mathrm{mL}^{-1}\right)\end{array}$ \\
& & constant $\left(\mathrm{K}_{\mathrm{D}}\right)$ & & $0.44 \pm 0.02$ \\
EndoMIPs IA & Langmuir & $5.24 \times 10^{-10} \mathrm{M}$ & 14.6 & $0.83 \pm 0.04$ \\
EndoMIPs MA & Langmuir & $4.40 \times 10^{-10} \mathrm{M}$ & 11.5 & $1.62 \pm 0.09$ \\
EndoMIPs AA & Langmuir & $1.63 \times 10^{-8} \mathrm{M}$ & 10.6 & \\
VancMIPs IA & Langmuir & $6.52 \times 10^{-6} \mathrm{M}$ & 13.1 & \\
& & & & \\
\hline
\end{tabular}

*The average yield of three batch productions determined for each monomer and the calculated values for limit of detection are tabulated. 
2016-06-21

Ultrasensitive detection of endotoxins using computationally designed nanoMIPs

Altintas, Zeynep

Elsevier

Zeynep Altintas, Mohammed J. Abdin, Alexander M. Tothill, Kal Karim, Ibtisam E. Tothill, Ultrasensitive detection of endotoxins using computationally designed nanoMIPs, Analytica Chimica Acta, Volume 935, 7 September 2016, pp239-248

http://dx.doi.org/10.1016/j.aca.2016.06.013

Downloaded from Cranfield Library Services E-Repository 